\title{
Panel test and chemical analyses of commercial olive oils: a comparative study
}

\author{
Simone Circi ${ }^{1}$, Donatella Capitani ${ }^{2}$, Antonio Randazzo ${ }^{3}$, Cinzia Ingallina' ${ }^{1}$ Luisa Mannina ${ }^{1,2^{*}}$ \\ and Anatoly P. Sobolev²
}

\begin{abstract}
Background: The quality grade of an olive oil is defined according to the results of analytical and organoleptic examinations.The increasing attention towards both olive oil quality and quality verification methods prompted us to undertake a "critical" analysis of analytical and sensory data supplied by an International Certificated Body (ICB), relative to commercial olive oils produced in Mediterranean areas and purchased in Italy and in USA.

Methods: ICB data included chemical analyses namely free acidity, peroxide index, spectrophotometric UV evaluation, fatty acid ethyl esters and stigmadiens content and organoleptic evaluations carried out by nine official International Olive Council labs according to EEC Regulation 2568/91.

Results: The results of the chemical analyses, except the fatty acid ethyl ester content, obtained from the nine labs were consistent giving rise to the same quality grade. In nearly all samples, the fatty acid ethyl ester content was close to the threshold established for extra virgin olive oils indicating a non-excellent quality of the olive oils. Organoleptic evaluations, commonly called panel test, given by the nine labs were not consistent.

Conclusions: The EEC Regulation 2568/91 does not give any indication on the way to report the uncertainty of the results, and in the case of extra virgin olive oils with a borderline value, the way to report the fatty acid ethyl ester content, with or without the uncertainty, can create confusion in defining the olive oil quality grade. Panel test seemed to work well only in the case of extremely good olive oils, whereas, in commercial extra virgin olive oils with borderline value of fatty acid ethyl ester content, a different sensory sensibility seems to be in the different IOC labs.
\end{abstract}

Keywords: Olive oil, Panel test (organoleptic evaluation), Chemical analyses, Fatty acid ethyl ester content

\section{Background}

EEC Regulation 2568/91 [1] and International Olive Council (IOC) [2] have established both analytical and organoleptic criteria to define the quality grade of an olive oil. According to the results of chemical and sensory analyses, an olive oil can be classified as extra virgin olive oil (EVOO), virgin olive oil or lampante olive oil. Each category has a completely different commercial and nutritional value. Extra virgin olive oil (EVOO) is a natural fruit juice with peculiar nutritional, healthy [3] and sensory [4] qualities as well as it represents a fundamental component of Mediterranean diet which is very

\footnotetext{
*Correspondence: luisa.mannina@uniroma1.it

1 Dipartimento di Chimica e Tecnologie del Farmaco, Sapienza Università di Roma, Rome, Italy

Full list of author information is available at the end of the article
}

rich in mono-unsaturated fatty acids [5] and polyphenols [6]. On the contrary, lampante olive oils have a distinctly unpleasant smell, are not edible and are used for the production of refined olive oils. It is well known that selling virgin olive oils or lampante olive oils as EVOOs is a fraud. However, another important aspect to consider is that the downgrading of an EVOO to a lower category implies a big economic loss for the producers. So, it is fundamental that both the chemical analyses and the organoleptic evaluations used to define quality grade are "sufficiently" objective and reproducible.

In this paper, a pilot analysis of data supplied by International Certificated Body, relative to 16 olive oils produced in the Mediterranean area, was carried out to verify reproducibility and consistency of chemical analyses and organoleptic evaluations and therefore to 
highlight possible problems relative to quality grade classification. ICB data, including chemical analyses and organoleptic evaluations, were produced by nine official IOC labs according to EEC Regulation 2568/91. The recent scandals involving Italian olive oils, such as the one revealed in the 'New York Times' [7], prompted us to include data from olive oils purchased not only in Italy, the largest consumer of EVOOs, but also in non-Mediterranean countries such as USA.

\section{Methods}

Olive oils (16 samples) of 2014/2015 harvest year were purchased in Italy and Miami (USA) by an International Certificated Body, a recognized EU company that deals with services of certification. ICB also verified the preparation of samples carried out according to EN ISO 5555 reported in EEC Regulation 2568/91, shipped olive oils to laboratories and released a report on all the activity. Samples were collected and labelled with an alphanumeric code without any reference to the origin country. Brands and the packaging places of olive oils were made purposely anonymous to avoid damage from a possible negative or defamatory propaganda.

Chemical analyses and organoleptic evaluation were carried out by nine recognized IOC laboratories, selected from the lists of IOC-recognized laboratories $[8,9]$, see Additional file 1: Table S1, located in countries with a well-recognized tradition in the production of olive oils such as Italy (5 labs in Abruzzo, Lazio, Liguria, Sicily and Veneto regions), Spain (2 labs), and Greece (1 lab). A Slovenian lab was also included as a reference for the Eastern countries.

Chemical analyses included the determination of free acidity, peroxide index, UV spectrophotometric evaluation (K 232, K 270, $\Delta K$ ), fatty acid ethyl esters (FAEE) and stigmadiens content. Stigmadiens content, a parameter closely related to the presence of refined oils rather than a quality marker, was also considered as genuineness index. All the analyses were carried out according to the EEC Regulation 2568/91 (Annex II, III, IX, XVII, XX). For the determination of free acidity and peroxide index, the Slovenian lab used IOC method (ISO 660:2009 and ISO 3960:2010, respectively).

According to the official method (EEC Regulation 2568/91, Annex XII), in each IOC lab, organoleptic evaluations were carried out by a group of $8-12$ professional tasters trained to recognize, describe and quantify basic taste and odour properties. Olive oils were described through positive ("fruity", "bitter" and "pungent") and negative (for instance "rancid", "fusty", "musty" and "winey") attributes.

Quality grade classifications of olive oils purchased in Italy and in USA were determined by our laboratory according to EEC Regulation 2568/91.

\section{Results and discussion}

Chemical analysis results regarding olive oils from Italy and USA are reported in Tables 1 and 2, respectively.

Quality grade classifications of olive oils purchased in Italy and in USA, reported in Tables 3 and 4, respectively, were determined according to EEC Regulation 2568/91 and will be discussed separately. Defects detected by panel test of olive oils purchased in Italy and Miami are reported in Additional files 2: Tables S2 and 3: Table S3, respectively, whereas positive attributes are reported in Additional file 4: Table S4. Classification percentages of the olive oils purchased in Italy and in USA are reported in Additional files 5: Tables S5 and 6: Table S6, respectively.

\section{Samples purchased in Italy}

In the case of olive oils purchased in Italy, samples turned out to be EVOOs or virgin olive oils from the chemical point of view (Tables 1, 3 and Additional file 5: Table S5). Five samples $\left(\mathrm{O}_{\mathrm{i}} 1, \mathrm{O}_{\mathrm{i}} 3, \mathrm{O}_{\mathrm{i}} 6, \mathrm{O}_{\mathrm{i}} 7\right.$ and $\left.\mathrm{O}_{\mathrm{i}} 8\right)$ were judged as EVOOs whereas samples $\mathrm{O}_{\mathrm{i}} 2, \mathrm{O}_{\mathrm{i}} 4$ and $\mathrm{O}_{\mathrm{i}} 5$ turned out to be EVOOs or virgin olive oils according to the specific lab. In particular, sample $\mathrm{O}_{\mathrm{i}} 2$ turned out to be EVOO according to the chemical analysis results provided by Sicilian and Venetian labs and virgin olive oil for Abruzzo, Slovenian and Ligurian labs, whereas sample $\mathrm{O}_{\mathrm{i}} 4$ turned out to be EVOO according to Abruzzo and Sicilian labs and virgin olive oil for Slovenian, Ligurian and Venetian labs. Sample $\mathrm{O}_{\mathrm{i}} 5$ was classified virgin olive oil according to all the labs except the Venetian one. The parameter responsible for the declassing of $\mathrm{O}_{i} 2, \mathrm{O}_{i}$ 4 and $\mathrm{O}_{\mathrm{i}} 5$ samples from EVOOs to not EVOOs was the content of FAEE. The importance of FAEE content to define the quality of an olive oil is well known $[10,11]$ : fatty acid ethyl esters are the products of the trans-esterification reaction between fatty acids and ethanol produced by bacteria fermentation that occurs when olives are of poor quality. Because of this narrow relationship between olive oil quality and FAEE content, the threshold for FAEE content in EVOOs has been decreased along the time, from $40 \mathrm{mg} / \mathrm{kg}$ in 2013/2014 harvest year down to $35 \mathrm{mg} / \mathrm{kg}$ in 2014/2015. Although this limit has been further reduced to $\leq 30 \mathrm{mg} / \mathrm{kg}$ in 2015, it has been set again to $35 \mathrm{mg} / \mathrm{kg}[12,13]$ on July 2016 by IOC and on September 2016 by EEC.

It is interesting to note, see Table 1 , that the FAEE content was reported by different IOC labs with or without uncertainty. For instance, IOC lab located in Veneto (Italy) never provided the uncertainty related to FAEE measurement. Sample $\mathrm{O}_{\mathrm{i}} 4$ with a FAEE content of $39.7 \mathrm{mg} / \mathrm{kg}$ reported without any uncertainty has to be classified as virgin olive oil, whereas $\mathrm{O}_{i} 1$ with a FAEE content of $28 \mathrm{mg} / \mathrm{kg}$ has to be classified as extra 
Table 1 Chemical analysis results performed by IOC laboratories on eight olive oils purchased in Italy

\begin{tabular}{|c|c|c|c|c|c|c|c|}
\hline & $\begin{array}{l}\text { Free acidity } \\
\text { (\% oleic acid) }\end{array}$ & $\begin{array}{l}\text { Peroxide index } \\
\left(\mathrm{meq}_{2} / \mathrm{kg}\right)\end{array}$ & K 232 & K 270 & $\Delta K$ & FAEE $(\mathrm{mg} / \mathrm{kg})$ & $\begin{array}{l}\text { Stigmadienes } \\
(\mathrm{mg} / \mathrm{kg})\end{array}$ \\
\hline \multicolumn{8}{|l|}{$\mathrm{O}_{\mathrm{i}} 1$} \\
\hline Greece & ${ }^{*}$ & / & / & / & / & / & / \\
\hline Slovenia & $0.26 \pm 0.03^{1}$ & $9.8 \pm 1.8^{2}$ & $1.80 \pm 0.04^{3}$ & $0.147 \pm 0.009^{4}$ & $-0.002 \pm 0.001^{5}$ & $30.2 \pm 4.2^{6}$ & $0.0491 \pm 0.0280$ \\
\hline Spain 1 & / & / & / & / & / & / & / \\
\hline Abruzzo (IT) & $0.23 \pm 0.02$ & $11.1 \pm 0.1$ & $1.79 \pm 0.04$ & $0.151 \pm 0.040$ & $-0.001 \pm 0.001$ & $29 \pm 1$ & $0.04 \pm 0.01$ \\
\hline Lazio (IT) & / & / & / & / & / & / & / \\
\hline Liguria (IT) & $0.2 \pm 0.1$ & $9 \pm 2$ & $1.88 \pm 0.16$ & $0.20 \pm 0.06$ & $0.00 \pm 0.01$ & $32 \pm 8$ & $0.06 \pm 0.01$ \\
\hline Sicily (IT) & $0.24 \pm 0.05$ & $11 \pm 3$ & $1.77 \pm 0.05$ & $0.14 \pm 0.02$ & $0.002 \pm 0.006$ & $28 \pm 8$ & $0.04 \pm 0.02$ \\
\hline Veneto (IT) & $0.28 \pm 0.06$ & $10.0 \pm 2.3$ & $2.12 \pm 0.39$ & $0.16 \pm 0.04$ & $0.002 \pm 0.002$ & 28 & 0.04 \\
\hline \multicolumn{8}{|l|}{$\mathrm{O}_{\mathrm{i}} 2$} \\
\hline Greece & / & / & / & / & / & / & / \\
\hline Slovenia & $0.35 \pm 0.30$ & $12.8 \pm 2.3$ & $2.07 \pm 0.04$ & $0.176 \pm 0.009$ & $0.001 \pm 0.001$ & $36.8 \pm 5.1$ & $0.0376 \pm 0.0088$ \\
\hline Spain 1 & / & / & / & / & / & / & / \\
\hline Abruzzo (IT) & $0.34 \pm 0.02$ & $13.5 \pm 0.1$ & $1.891 \pm 0.004$ & $0.160 \pm 0.004$ & $0.0040 \pm 0.0001$ & $37 \pm 1$ & $0.03 \pm 0.01$ \\
\hline Lazio (IT) & / & / & / & / & / & / & / \\
\hline Liguria (IT) & $0.3 \pm 0.1$ & $13 \pm 2$ & $1.96 \pm 0.17$ & $0.19 \pm 0.06$ & $0.00 \pm 0.01$ & $38 \pm 10$ & $0.03 \pm 0.01$ \\
\hline Sicily (IT) & $0.36 \pm 0.05$ & $13 \pm 3$ & $1.95 \pm 0.05$ & $0.21 \pm 0.02$ & $0.0000 \pm 0.0006$ & $30 \pm 9$ & $0.05 \pm 0.02$ \\
\hline Veneto (IT) & $0.35 \pm 0.06$ & $13.0 \pm 2.3$ & $2.04 \pm 0.39$ & $0.17 \pm 0.04$ & $0.001 \pm 0.002$ & 33 & 0.02 \\
\hline \multicolumn{8}{|l|}{$\mathrm{O}_{\mathrm{i}} 3$} \\
\hline Greece & / & / & / & / & / & / & / \\
\hline Slovenia & $0.24 \pm 0.03$ & $7.3 \pm 1.3$ & $1.78 \pm 0.04$ & $0.201 \pm 0.009$ & $0.004 \pm 0.001$ & $24.6 \pm 3.4$ & $0.0238 \pm 0.0068$ \\
\hline Spain 1 & / & / & / & / & / & / & / \\
\hline Abruzzo (IT) & $0.23 \pm 0.02$ & $9.4 \pm 0.1$ & $1.615 \pm 0.004$ & $0.168 \pm 0.004$ & $0.0090 \pm 0.0001$ & $25 \pm 1$ & $0.01 \pm 0.01$ \\
\hline Lazio (IT) & / & / & / & / & / & / & / \\
\hline Liguria (IT) & $0.2 \pm 0.1$ & $8 \pm 2$ & $1.74 \pm 0.15$ & $0.20 \pm 0.06$ & $0.00 \pm 0.01$ & $27 \pm 7$ & $0.01 \pm 0.01$ \\
\hline Sicily (IT) & $0.23 \pm 0.05$ & $8 \pm 3$ & $1.60 \pm 0.05$ & $0.17 \pm 0.02$ & $0.004 \pm 0.006$ & $20 \pm 6$ & $0.05 \pm 0.02$ \\
\hline Veneto (IT) & $0.24 \pm 0.06$ & $8.0 \pm 2.3$ & $1.82 \pm 0.39$ & $0.18 \pm 0.04$ & $0.004 \pm 0.002$ & 23.7 & 0.03 \\
\hline \multicolumn{8}{|l|}{$\mathrm{O}_{\mathrm{i}} 4$} \\
\hline Greece & / & / & / & / & / & / & / \\
\hline Slovenia & $0.28 \pm 0.03$ & $6.2 \pm 1.1$ & $1.79 \pm 0.04$ & $0.122 \pm 0.009$ & $0.000 \pm 0.001$ & $35.1 \pm 4.9$ & $<0.01$ \\
\hline Spain 1 & / & / & / & / & / & / & / \\
\hline Abruzzo (IT) & $0.28 \pm 0.02$ & $7.9 \pm 0.1$ & $1.760 \pm 0.004$ & $0.117 \pm 0.004$ & $0.000 \pm 0.001$ & $35 \pm 1$ & $0.03 \pm 0.01$ \\
\hline Lazio (IT) & / & / & / & / & / & / & / \\
\hline Liguria (IT) & $0.3 \pm 0.1$ & $8 \pm 2$ & $1.84 \pm 0.16$ & $0.13 \pm 0.04$ & $0.00 \pm 0.01$ & $40 \pm 12$ & $0.01 \pm 0.01$ \\
\hline Sicily (IT) & $0.27 \pm 0.05$ & $8 \pm 3$ & $1.78 \pm 0.05$ & $0.12 \pm 0.02$ & $0.004 \pm 0.006$ & $33 \pm 9$ & $0.05 \pm 0.02$ \\
\hline Veneto (IT) & $0.26 \pm 0.06$ & $7.0 \pm 2.3$ & $1.98 \pm 0.39$ & $0.13 \pm 0.04$ & $0.001 \pm 0.002$ & 39.7 & 0.02 \\
\hline \multicolumn{8}{|l|}{$\mathrm{O}_{i} 5$} \\
\hline Greece & / & / & / & / & / & / & / \\
\hline Slovenia & $0.39 \pm 0.03$ & $8.2 \pm 1.5$ & $1.86 \pm 0.04$ & $0.140 \pm 0.009$ & $-0.002 \pm 0.001$ & $35.5 \pm 5.0$ & $0.0136 \pm 0.0025$ \\
\hline Spain 1 & / & / & / & / & / & / & / \\
\hline Abruzzo (IT) & $0.36 \pm 0.02$ & $9.1 \pm 0.1$ & $1.812 \pm 0.004$ & $0.138 \pm 0.004$ & $-0.001 \pm 0.001$ & $36 \pm 1$ & $0.02 \pm 0.01$ \\
\hline Lazio (IT) & / & / & / & / & / & / & / \\
\hline Liguria (IT) & $0.4 \pm 0.1$ & $8 \pm 2$ & $1.96 \pm 0.17$ & $0.16 \pm 0.05$ & $0.00 \pm 0.01$ & $39 \pm 10$ & $0.01 \pm 0.01$ \\
\hline Sicily (IT) & $0.36 \pm 0.05$ & $9 \pm 3$ & $1.69 \pm 0.05$ & $0.12 \pm 0.02$ & $0.001 \pm 0.006$ & $36 \pm 10$ & $0.04 \pm 0.02$ \\
\hline Veneto (IT) & $0.38 \pm 0.06$ & $8.0 \pm 2.3$ & $1.96 \pm 0.39$ & $0.16 \pm 0.04$ & $0.002 \pm 0.002$ & 35 & 0.04 \\
\hline \multicolumn{8}{|l|}{$O_{i} 6$} \\
\hline Greece & / & / & / & / & / & / & / \\
\hline Slovenia & $0.19 \pm 0.03$ & $8.9 \pm 1.6$ & $2.21 \pm 0.04$ & $0.149 \pm 0.009$ & $-0.001 \pm 0.001$ & $11.7 \pm 1.6$ & $0.0129 \pm 0.0023$ \\
\hline Spain 1 & / & / & / & / & / & / & / \\
\hline
\end{tabular}


Table 1 continued

\begin{tabular}{|c|c|c|c|c|c|c|c|}
\hline & $\begin{array}{l}\text { Free acidity } \\
\text { (\% oleic acid) }\end{array}$ & 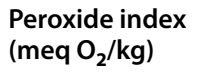 & K 232 & K 270 & $\Delta K$ & $\mathrm{FAEE}(\mathrm{mg} / \mathrm{kg})$ & $\begin{array}{l}\text { Stigmadienes } \\
(\mathrm{mg} / \mathrm{kg})\end{array}$ \\
\hline Abruzzo (IT) & $0.16 \pm 0.02$ & $10.2 \pm 0.1$ & $2.089 \pm 0.004$ & $0.136 \pm 0.004$ & $-0.001 \pm 0.001$ & $14 \pm 1$ & $0.02 \pm 0.01$ \\
\hline Lazio (IT) & / & / & / & / & / & / & / \\
\hline Liguria (IT) & $0.2 \pm 0.1$ & $9 \pm 2$ & $2.16 \pm 0.19$ & $0.17 \pm 0.06$ & $0.00 \pm 0.01$ & $10 \pm 3$ & $<0.01 \pm 0.01$ \\
\hline Sicily (IT) & $0.16 \pm 0.05$ & $11 \pm 3$ & $2.12 \pm 0.05$ & $0.16 \pm 0.02$ & $0.000 \pm 0.006$ & $10 \pm 4$ & $0.03 \pm 0.02$ \\
\hline Veneto (IT) & $0.18 \pm 0.06$ & $9.0 \pm 2.3$ & $2.26 \pm 0.39$ & $0.16 \pm 0.04$ & $0.002 \pm 0.002$ & 11 & 0.02 \\
\hline \multicolumn{8}{|l|}{$\mathrm{O}_{\mathrm{i}} 7$} \\
\hline Greece & / & / & / & / & / & / & / \\
\hline Slovenia & $0.23 \pm 0.03$ & $7.9 \pm 1.4$ & $1.86 \pm 0.04$ & $0.126 \pm 0.009$ & $-0.003 \pm 0.001$ & $24.4 \pm 3.4$ & $0.0176 \pm 0.0032$ \\
\hline Spain 1 & / & / & / & / & / & / & / \\
\hline Abruzzo (IT) & $0.23 \pm 0.02$ & $9.7 \pm 1.0$ & $1.807 \pm 0.004$ & $0.126 \pm 0.004$ & $-0.003 \pm 0.001$ & $30 \pm 1$ & $0.02 \pm 0.01$ \\
\hline Lazio (IT) & / & / & / & / & / & / & / \\
\hline Liguria (IT) & $0.2 \pm 0.1$ & $8 \pm 2$ & $1.87 \pm 0.16$ & $0.15 \pm 0.05$ & $0.00 \pm 0.01$ & $32 \pm 8$ & $0.01 \pm 0.01$ \\
\hline Sicily (IT) & $0.22 \pm 0.05$ & $8 \pm 3$ & $1.87 \pm 0.05$ & $0.15 \pm 0.02$ & $0.003 \pm 0.006$ & $20 \pm 6$ & $0.02 \pm 0.02$ \\
\hline Veneto (IT) & $0.25 \pm 0.06$ & $9.0 \pm 2.3$ & $1.99 \pm 0.39$ & $0.14 \pm 0.04$ & $0.004 \pm 0.002$ & 22 & 0.04 \\
\hline \multicolumn{8}{|l|}{$\mathrm{O}_{i} 8$} \\
\hline Greece & / & / & / & / & / & / & / \\
\hline Slovenia & $0.31 \pm 0.03$ & $11.6 \pm 2.1$ & $2.38 \pm 0.04$ & $0.152 \pm 0.009$ & $-0.002 \pm 0.001$ & $29.0 \pm 4.1$ & $0.0161 \pm 0.0300$ \\
\hline Spain 1 & / & / & / & / & / & / & / \\
\hline Abruzzo (IT) & $0.32 \pm 0.01$ & $13.1 \pm 0.1$ & $2.049 \pm 0.004$ & $0.143 \pm 0.004$ & $-0.002 \pm 0.001$ & $33 \pm 1$ & $0.03 \pm 0.01$ \\
\hline Lazio (IT) & / & / & / & / & / & / & / \\
\hline Liguria (IT) & $0.3 \pm 0.1$ & $11 \pm 2$ & $2.57 \pm 0.22$ & $0.18 \pm 0.06$ & $0.00 \pm 0.01$ & $31 \pm 8$ & $0.01 \pm 0.01$ \\
\hline Sicily (IT) & $0.32 \pm 0.05$ & $13 \pm 3$ & $2.35 \pm 0.05$ & $0.15 \pm 0.02$ & $0.002 \pm 0.006$ & $25 \pm 7$ & $0.04 \pm 0.02$ \\
\hline Veneto (IT) & $0.33 \pm 0.06$ & $13.0 \pm 2.3$ & $2.49 \pm 0.39$ & $0.16 \pm 0.04$ & $0.003 \pm 0.002$ & 27 & 0.04 \\
\hline
\end{tabular}

$/{ }^{*}$ Analysis was not performed

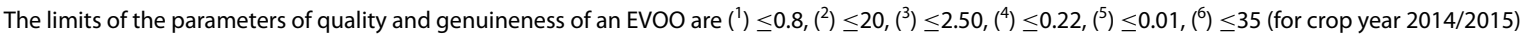

The limits of the parameters of a virgin olive oil are $2 \leq\left(^{1}\right) \leq 0.8,\left({ }^{2}\right) \leq 20,\left({ }^{3}\right) \leq 2.60,\left(\left(^{4}\right) \leq 0.25,\left({ }^{5}\right) \leq 0.01\right.$

virgin olive oil. On the other hand, some labs reported a value higher than $35 \mathrm{mg} / \mathrm{kg}$ (the threshold value for year 2014/2015) with the threshold value within the uncertainty, see for example the results of Slovenian lab reported for sample $\mathrm{O}_{\mathrm{i}} 2$ with a FAEE content of $36.8 \pm 5.1 \mathrm{mg} / \mathrm{kg}$. Finally, other labs reported a value lower than $35 \mathrm{mg} / \mathrm{kg}$ with the threshold within the uncertainty, see for example the results of Ligurian lab for sample $\mathrm{O}_{\mathrm{i}} 1$ with a FAEE content of $32 \pm 8 \mathrm{mg} / \mathrm{kg}$. How to use these data to classify olive oils? A strict interpretation of EEC Regulation 2568/91 that does not give any indication on the way to report the uncertainty of the results implies that any olive oil with a FAEE content value above the threshold has to be declassified from extra virgin olive oil to not extra virgin olive. Therefore, $\mathrm{O}_{\mathrm{i}} 2$ with a FAEE content of $36.8 \pm 5.1 \mathrm{mg} / \mathrm{kg}$ has to be classified as not extra virgin olive oil, whereas $\mathrm{O}_{\mathrm{i}} 1$ with a FAEE content of $32 \pm 8 \mathrm{mg} / \mathrm{kg}$ has to be classified as extra virgin olive oil. Whereas an analytical point of view to report a result with the uncertainty is a correct way, to consider the uncertainty for the olive oil classification can give rise to confusion. In fact, if the uncertainty is considered, these two olive oils should have the same grade, i.e. extra virgin olive oil. On the other hand, taking into account the upper limit of uncertainty, an olive oil with a value of $32 \pm 8 \mathrm{mg} / \mathrm{kg}$ could be considered not extra virgin. Therefore, the way to report the FAEE content with the uncertainty can create confusion, when the olive oil commercial classification is required.

Organoleptic evaluation carried out from the different labs were extremely controversial. For instance, all the olive oils were judged as EVOOs according to the results of the Sicilian lab, whereas no sample was judged as EVOO according to the results of the Greek lab. These results clearly show that the same olive oil can be judged EVOO, virgin olive oil or lampante olive oil according to the the lab. This is the case of samples $\mathrm{O}_{\mathrm{i}} 2, \mathrm{O}_{\mathrm{i}} 3, \mathrm{O}_{\mathrm{i}} 4$, $\mathrm{O}_{\mathrm{i}} 5$, whereas samples $\mathrm{O}_{\mathrm{i}} 1, \mathrm{O}_{\mathrm{i}} 6, \mathrm{O}_{\mathrm{i}} 7, \mathrm{O}_{\mathrm{i}} 8$ were judged virgin olive oils or EVOOs. Only sample $\mathrm{O}_{\mathrm{i}} 6$ was judged EVOO according to the results of all the labs except the Greek one. It is important to note that this olive oil has a FAEE concentration $<15 \mathrm{mg} / \mathrm{kg}$ well below the threshold 
Table 2 Chemical analysis results performed by IOC laboratories on eight olive oils purchased in USA (Miami)

\begin{tabular}{|c|c|c|c|c|c|c|c|}
\hline & $\begin{array}{l}\text { Free acidity } \\
\text { (\% oleic acid) }\end{array}$ & $\begin{array}{l}\text { Peroxide index } \\
\left(\mathrm{meq} \mathrm{O}_{2} / \mathrm{kg}\right)\end{array}$ & K 232 & K 270 & $\Delta K$ & $\mathrm{FAEE}(\mathrm{mg} / \mathrm{kg})$ & Stigmadienes $(\mathrm{mg} / \mathrm{kg})$ \\
\hline \multicolumn{8}{|l|}{$\mathrm{O}_{u} 1$} \\
\hline Greece & ${ }^{*}$ & / & / & / & / & / & / \\
\hline Slovenia & $0.41 \pm 0.03$ & $9.1 \pm 1.6$ & $2.17 \pm 0.04$ & $0.130 \pm 0.009$ & $-0.002 \pm 0.001$ & $34.5 \pm 4.8$ & $0.0117 \pm 0.0021$ \\
\hline Spain 1 & / & / & / & / & / & / & / \\
\hline Spain 2 & 0.39 & 10.60 & 2.27 & 0.13 & $<0.01$ & 38.80 & $<0.05$ \\
\hline Lazio (IT) & / & / & / & / & / & / & / \\
\hline $\begin{array}{l}\text { Liguria } \\
\text { (IT) }\end{array}$ & $0.4 \pm 0.1$ & $10 \pm 2$ & $2.32 \pm 0.20$ & $0.14 \pm 0.04$ & $0.00 \pm 0.01$ & $35 \pm 9$ & $<0.01 \pm 0.01$ \\
\hline Sicily (IT) & $0.40 \pm 0.05$ & $10 \pm 3$ & $2.04 \pm 0.05$ & $0.09 \pm 0.02$ & $0.002 \pm 0.006$ & $37 \pm 10$ & $0.01 \pm 0.02$ \\
\hline $\begin{array}{l}\text { Veneto } \\
\text { (IT) }\end{array}$ & $0.40 \pm 0.06$ & $10.0 \pm 2.3$ & $2.45 \pm 0.39$ & $0.15 \pm 0.04$ & $0.003 \pm 0.002$ & 34 & 0.02 \\
\hline \multicolumn{8}{|l|}{$\mathrm{O}_{\mathrm{u}} 2$} \\
\hline Greece & / & / & / & / & / & / & / \\
\hline Slovenia & $0.27 \pm 0.03$ & $16.0 \pm 2.9$ & $2.79 \pm 0.04$ & $0.225 \pm 0.009$ & $0.004 \pm 0.001$ & $<15$ & $0.089 \pm 0.016$ \\
\hline Spain 1 & / & / & / & / & / & / & / \\
\hline Spain 2 & / & / & / & / & / & / & / \\
\hline Lazio (IT) & / & / & / & / & / & / & / \\
\hline $\begin{array}{l}\text { Liguria } \\
\text { (IT) }\end{array}$ & $0.3 \pm 0.1$ & $18 \pm 2$ & $2.98 \pm 0.26$ & $0.23 \pm 0.07$ & $0.00 \pm 0.01$ & $22 \pm 6$ & $0.10 \pm 0.02$ \\
\hline Sicily (IT) & $0.28 \pm 0.05$ & $20 \pm 3$ & $2.76 \pm 0.05$ & $0.24 \pm 0.02$ & $0.002 \pm 0.006$ & $22 \pm 7$ & $0.10 \pm 0.03$ \\
\hline $\begin{array}{l}\text { Veneto } \\
\text { (IT) }\end{array}$ & $0.27 \pm 0.06$ & $17.8 \pm 2.3$ & $3.08 \pm 0.39$ & $0.24 \pm 0.04$ & $0.002 \pm 0.002$ & 15 & 0.06 \\
\hline \multicolumn{8}{|l|}{$\mathrm{O}_{\mathrm{u}} 3$} \\
\hline Greece & / & / & / & / & / & / & / \\
\hline Slovenia & $0.41 \pm 0.03$ & $12.0 \pm 2.2$ & $2.84 \pm 0.04$ & $0.266 \pm 0.009$ & $0.006 \pm 0.001$ & $18.6 \pm 2.6$ & $0.0392 \pm 0.0071$ \\
\hline Spain 1 & / & / & / & / & / & / & / \\
\hline Spain 2 & 0.41 & 16.90 & 2.71 & 0.28 & 0.01 & 27.60 & $<0.05$ \\
\hline Lazio (IT) & / & / & / & / & / & / & / \\
\hline $\begin{array}{l}\text { Liguria } \\
\text { (IT) }\end{array}$ & $0.4 \pm 0.1$ & $14 \pm 2$ & $2.98 \pm 0.26$ & $0.28 \pm 0.09$ & $0.00 \pm 0.01$ & $25 \pm 6$ & $0.05 \pm 0.01$ \\
\hline Sicily (IT) & $0.43 \pm 0.05$ & $16 \pm 3$ & $2.79 \pm 0.05$ & $0.29 \pm 0.02$ & $0.006 \pm 0.006$ & $28 \pm 8$ & $0.05 \pm 0.02$ \\
\hline $\begin{array}{l}\text { Veneto } \\
\text { (IT) }\end{array}$ & $0.41 \pm 0.06$ & $14.6 \pm 2.3$ & $3.02 \pm 0.39$ & $0.30 \pm 0.04$ & $0.005 \pm 0.002$ & 28 & 0.06 \\
\hline \multicolumn{8}{|l|}{$\mathrm{O}_{\mathrm{u}} 4$} \\
\hline Greece & / & / & / & / & / & / & / \\
\hline Slovenia & $0.21 \pm 0.03$ & $7.9 \pm 1.4$ & $1.90 \pm 0.04$ & $0.140 \pm 0.009$ & $-0.001 \pm 0.001$ & $39.0 \pm 5.5$ & $0.0162 \pm 0.0029$ \\
\hline Spain 1 & / & / & / & / & / & / & / \\
\hline Spain 2 & 0.21 & 8.80 & 2.05 & 0.14 & $<0.01$ & 47.80 & $<0.05$ \\
\hline Lazio (IT) & / & / & / & / & / & / & / \\
\hline $\begin{array}{l}\text { Liguria } \\
\text { (IT) }\end{array}$ & $0.2 \pm 0.1$ & $8 \pm 2$ & $2.00 \pm 0.17$ & $0.16 \pm 0.05$ & $0.00 \pm 0.01$ & $45 \pm 11$ & $0.01 \pm 0.01$ \\
\hline Sicily (IT) & $0.21 \pm 0.05$ & $10 \pm 3$ & $1.85 \pm 0.05$ & $0.14 \pm 0.02$ & $0.007 \pm 0.006$ & $49 \pm 13$ & $0.02 \pm 0.02$ \\
\hline $\begin{array}{l}\text { Veneto } \\
\text { (IT) }\end{array}$ & $0.21 \pm 0.06$ & $8.3 \pm 2.3$ & $2.12 \pm 0.39$ & $0.18 \pm 0.04$ & $0.002 \pm 0.002$ & 46 & 0.05 \\
\hline \multicolumn{8}{|l|}{$\mathrm{O}_{\mathrm{u}} 5$} \\
\hline Greece & / & / & / & / & / & / & / \\
\hline Slovenia & $0.21 \pm 0.03$ & $8.6 \pm 1.6$ & $1.92 \pm 0.04$ & $0.238 \pm 0.009$ & $0.007 \pm 0.001$ & $39.5 \pm 5.5$ & $0.0331 \pm 0.0060$ \\
\hline Spain 1 & / & / & / & / & / & / & / \\
\hline Spain 2 & 0.20 & 9.60 & 2.04 & 0.24 & 0.01 & 43.20 & $<0.05$ \\
\hline Lazio (IT) & / & / & / & / & / & / & / \\
\hline
\end{tabular}


Table 2 continued

\begin{tabular}{|c|c|c|c|c|c|c|c|}
\hline & $\begin{array}{l}\text { Free acidity } \\
\text { (\% oleic acid) }\end{array}$ & $\begin{array}{l}\text { Peroxide index } \\
\text { (meq } \mathrm{O}_{2} / \mathrm{kg} \text { ) }\end{array}$ & K 232 & K 270 & $\Delta K$ & FAEE (mg/kg) & Stigmadienes $(\mathrm{mg} / \mathrm{kg})$ \\
\hline $\begin{array}{l}\text { Liguria } \\
\text { (IT) }\end{array}$ & $0.3 \pm 0.1$ & $9 \pm 2$ & $2.16 \pm 0.19$ & $0.27 \pm 0.08$ & $0.01 \pm 0.01$ & $43 \pm 11$ & $0.03 \pm 0.01$ \\
\hline Sicily (IT) & $0.22 \pm 0.05$ & $10 \pm 3$ & $1.99 \pm 0.05$ & $0.26 \pm 0.02$ & $0.007 \pm 0.006$ & $48 \pm 13$ & $0.03 \pm 0.02$ \\
\hline $\begin{array}{l}\text { Veneto } \\
\text { (IT) }\end{array}$ & $0.22 \pm 0.06$ & $8.7 \pm 2.3$ & $2.10 \pm 0.39$ & $0.25 \pm 0.04$ & $0.006 \pm 0.002$ & 38 & 0.03 \\
\hline \multicolumn{8}{|l|}{$\mathrm{O}_{u} 6$} \\
\hline Greece & / & / & / & / & / & / & / \\
\hline Slovenia & $0.25 \pm 0.03$ & $9.9 \pm 1.8$ & $2.00 \pm 0.04$ & $0.129 \pm 0.009$ & $-0.003 \pm 0.001$ & $29.7 \pm 4.2$ & $0.0084 \pm 0.0015$ \\
\hline Spain 1 & / & / & / & / & / & / & / \\
\hline Spain 2 & 0.23 & 13.20 & 2.04 & 0.13 & $<0.01$ & 35.00 & $<0.05$ \\
\hline Lazio (IT) & / & / & / & / & / & / & / \\
\hline $\begin{array}{l}\text { Liguria } \\
\text { (IT) }\end{array}$ & $0.30 \pm 0.01$ & $11 \pm 2$ & $2.03 \pm 0.18$ & $0.14 \pm 0.05$ & $0.00 \pm 0.01$ & $32 \pm 8$ & $0.01 \pm 0.01$ \\
\hline Sicily (IT) & $0.39 \pm 0.05$ & $9 \pm 3$ & $1.93 \pm 0.05$ & $0.13 \pm 0.02$ & $0.003 \pm 0.006$ & $34 \pm 9$ & $0.01 \pm 0.02$ \\
\hline $\begin{array}{l}\text { Veneto } \\
\text { (IT) }\end{array}$ & $0.24 \pm 0.06$ & $10.9 \pm 2.3$ & $2.14 \pm 0.39$ & $0.15 \pm 0.04$ & $0.004 \pm 0.002$ & 29 & 0.05 \\
\hline \multicolumn{8}{|l|}{$\mathrm{O}_{\mathrm{u}} 7$} \\
\hline Greece & / & / & / & / & / & / & / \\
\hline Slovenia & $0.27 \pm 0.03$ & $10.0 \pm 1.8$ & $1.95 \pm 0.04$ & $0.148 \pm 0.009$ & $-0.001 \pm 0.001$ & $63.8 \pm 8.9$ & $0.0257 \pm 0.0046$ \\
\hline Spain 1 & / & / & / & / & / & / & / \\
\hline Spain 2 & 0.26 & 11.80 & 1.99 & 0.14 & $<0.01$ & 67.40 & $<0.05$ \\
\hline Lazio (IT) & / & / & / & / & / & / & / \\
\hline $\begin{array}{l}\text { Liguria } \\
\text { (IT) }\end{array}$ & $0.3 \pm 0.1$ & $10 \pm 2$ & $2.07 \pm 0.18$ & $0.14 \pm 0.05$ & $0.00 \pm 0.01$ & $65 \pm 17$ & $0.01 \pm 0.01$ \\
\hline Sicily (IT) & $0.27 \pm 0.05$ & $12 \pm 3$ & $1.95 \pm 0.05$ & $0.15 \pm 0.02$ & $0.001 \pm 0.006$ & $72 \pm 18$ & $0.01 \pm 0.02$ \\
\hline $\begin{array}{l}\text { Veneto } \\
\text { (IT) }\end{array}$ & $0.26 \pm 0.06$ & $10.7 \pm 2.3$ & $2.17 \pm 0.39$ & $0.16 \pm 0.04$ & $0.002 \pm 0.002$ & 60 & 0.02 \\
\hline \multicolumn{8}{|l|}{$\mathrm{O}_{u} 8$} \\
\hline Greece & / & / & / & / & / & / & / \\
\hline Slovenia & $0.32 \pm 0.03$ & $8.0 \pm 1.4$ & $2.01 \pm 0.04$ & $0.145 \pm 0.009$ & $-0.002 \pm 0.001$ & $29.8 \pm 4.2$ & $<0.01$ \\
\hline Spain 1 & / & / & / & / & / & / & / \\
\hline Spain 2 & 0.30 & 12.00 & 2.16 & 0.15 & $<0.01$ & 33.20 & $<0.05$ \\
\hline Lazio (IT) & / & / & / & / & / & / & / \\
\hline $\begin{array}{l}\text { Liguria } \\
\text { (IT) }\end{array}$ & $0.3 \pm 0.1$ & $9 \pm 2$ & $2.15 \pm 0.19$ & $0.15 \pm 0.04$ & $0.00 \pm 0.01$ & $29 \pm 8$ & $0.01 \pm 0.01$ \\
\hline Sicily (IT) & $0.31 \pm 0.05$ & $8 \pm 3$ & $2.03 \pm 0.05$ & $0.16 \pm 0.02$ & $0.002 \pm 0.006$ & $32 \pm 9$ & $0.01 \pm 0.02$ \\
\hline $\begin{array}{l}\text { Veneto } \\
\text { (IT) }\end{array}$ & $0.29 \pm 0.06$ & $10.1 \pm 2.3$ & $2.20 \pm 0.39$ & $0.16 \pm 0.04$ & $0.003 \pm 0.002$ & 28 & 0.04 \\
\hline
\end{tabular}

$1{ }^{*}$ Analysis was not performed

of $35 \mathrm{mg} / \mathrm{kg}$, whereas the other olive oils exhibit a FAEE content which is slightly less or higher than the threshold value. The strict connection between FAEE content and the positive attributes by organoleptic evaluation of an olive oil has been previously reported $[10,14]$.

Therefore, all the obtained results seem to suggest that only in the case of olive oils with extremely "good" chemical analyses, including a low FAEE content (neither borderline nor higher than the threshold value), the organoleptic evaluation can be reproducible.

\section{Samples purchased in USA}

Olive oils purchased in Miami (USA) were judged, see Tables 2 and 4 and Additional file 6: Table S6, EVOOs $\left(\mathrm{O}_{u} 6\right.$ and $\left.\mathrm{O}_{u} 8\right)$, virgin olive oils $\left(\mathrm{O}_{u} 4, \mathrm{O}_{u} 5\right.$ and $\left.\mathrm{O}_{u} 7\right)$ and lampante olive oils $\left(\mathrm{O}_{\mathrm{u}} 2\right.$ and $\left.\mathrm{O}_{\mathrm{u}} 3\right)$ according to the chemical analysis results. The judgment given by the different labs for these five samples was univocal: samples $\mathrm{O}_{\mathrm{u}} 2$ and $\mathrm{O}_{\mathrm{u}} 3$ turned out to be lampante olive oils because of their high $\mathrm{K} 232$ value $(>2.60)$ whereas samples $\mathrm{O}_{\mathrm{u}} 4$ and $\mathrm{O}_{\mathrm{u}} 7$ were classified as virgin olive oil due to 


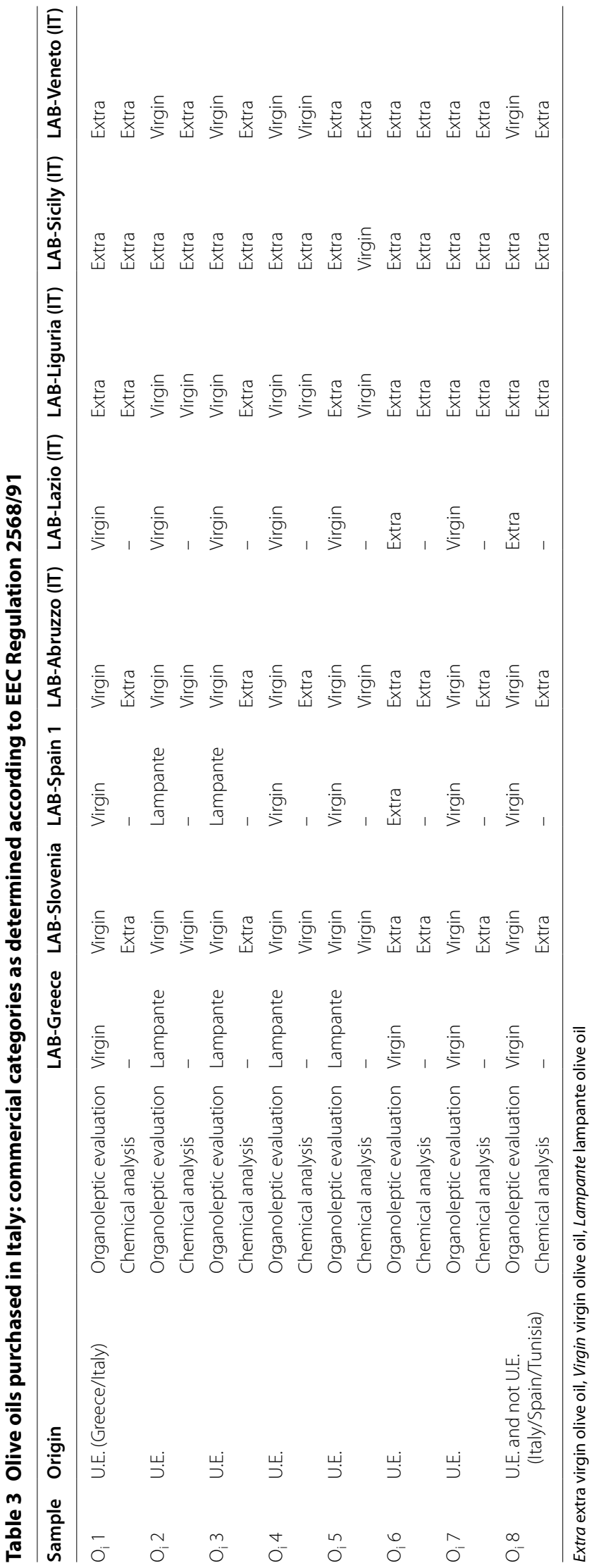




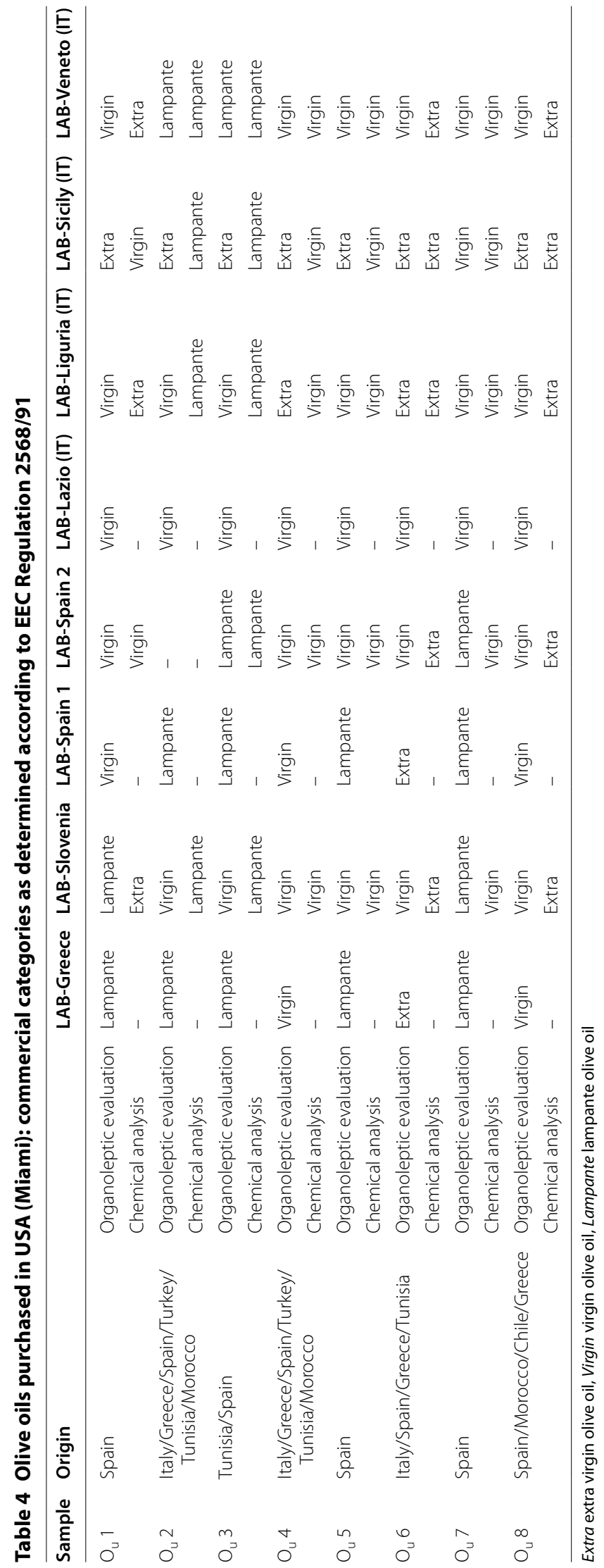


the high FAEE content ( $>35 \mathrm{mg} / \mathrm{kg}$ ). Sample $\mathrm{O}_{\mathrm{u}} 5$ turned out to be virgin olive oil because of the high content of FAEE $(>35 \mathrm{mg} / \mathrm{kg})$ in the case of Ligurian and Venetian labs or for both K 270 value $(0.22<\mathrm{K} 270<0.25)$ and FAEE content in the case of Slovenian, Sicilian and Spain 2 labs.

Finally, samples $\mathrm{O}_{\mathrm{u}} 1$ and $\mathrm{O}_{\mathrm{u}} 4$ were judged virgin olive oils or EVOOs according to the labs results: the critical point was again the FAEE content given without error or with a high error.

The results of organoleptic evaluation given by the different laboratories turned out to be in disagreement. For instance, all samples were judged virgin olive oils due to a median of defects $>0$ according to the Lazio lab results, whereas all samples, except one, were judged EVOOs according to the Sicilian lab results. Samples $\mathrm{O}_{u} 1, \mathrm{O}_{u}$ 2, $\mathrm{O}_{\mathrm{u}} 3$ and $\mathrm{O}_{\mathrm{u}} 5$ turned out to be lampante olive oils, virgin olive oils or EVOOs according to the lab, whereas sample $\mathrm{O}_{\mathrm{u}} 7$ was judged lampante olive oil or virgin olive oil. Finally, samples $\mathrm{O}_{u} 4, \mathrm{O}_{\mathrm{u}} 6$ and $\mathrm{O}_{\mathrm{u}} 8$ were judged virgin olive oils or EVOOs according to the labs.

Again, this disagreement of sensory evaluation can be related to some borderline value in the chemical parameters. It is interesting to note that the only two samples, $\mathrm{O}_{\mathrm{u}}$ 6 and $\mathrm{O}_{\mathrm{u}}$ 8, judged EVOOs by the chemical analysis, with a slightly less FAEE content than the threshold value were classified as EVOOs or virgin olive oils by the organoleptic evaluation. A similar case is also represented by the sample $\mathrm{O}_{\mathrm{u}} 1$ which, through chemical analyses, was judged EVOO in three cases out of five with a borderline FAEE content, whereas, through organoleptic evaluation, it was classified as EVOO, virgin olive oil or even lampante olive oil.

\section{Conclusions}

The results reported in this paper highlight some important aspects to be considered in the olive oil commercial classification.

First of all, the non-uniform way to report the FAEE content with or without uncertainty can create confusion in the quality grade classification, especially in case of borderline values of FAEE content. It is important to underline that the EEC Regulation 2568/91 does not give any indication on the way to report the uncertainty of the results.

Our data analysis underlines that it is crucial to find out what causes the variability of judgement in the organoleptic evaluation. Sensory panel test seems to work well in the case of extremely good olive oils, whereas in the case of common commercial EVOOs can give discordant results. A different sensory sensibility seems to be present in the different IOC panel labs, especially in case of EVOOs characterized by a FAEE content very close to the threshold. For a given olive oil, the question to be or not to be classified as EVOO becomes a question strictly linked to the lab.

In our opinion, organoleptic evaluation is extremely important for a global picture of the sensory properties of olive oil and it is particularly precious and not replaceable with other analysis to give add values to PDO or other peculiar EVOOs. On the other hand, this analysis seems to be not enough reproducible in the case of common commercial EVOOs probably due to different sensory sensibility in the different IOC panel labs and therefore it is not suitable for a legally accepted official evaluation.

\section{Additional files}

Additional file 1: Table S1. IOC laboratories chosen to carry out chemical analyses and organoleptic evaluation.

Additional file 2: Table S2. Defects, resulting by organoleptic evaluation performed by the eight IOC laboratories, of the eight olive oils purchased in Italy. The median values are reported for each defect. In certain cases, some laboratories provided only the predominant defect of an olive oil despite the detection of more defects. Labs of Spain 1 and Greece just supplied the median of the predominant defect without further details.

Additional file 3: Table S3. Defects, resulting by organoleptic evaluation performed by 8 IOC laboratories, of the eight olive oils purchased in USA (Miami). The median values are reported for each defect. In certain cases, some laboratories provided only the predominant defect of an olive oil despite the detection of more defects. Labs of Spain 2 and Greece just supplied the median of the predominant defect without further details. * Sample $\mathrm{O}_{\mathrm{u}} 2$ was not analyzed by Spain 2 lab.

Additional file 4: Table S4. Positive attributes, resulting by organoleptic evaluation performed by 8 IOC laboratories, of the eight olive oils purchased in Italy and the eight olive oils purchased in USA (Miami). The median values are reported for each defect. Results of "Bitter" and "Pungent" attributes were not provided by all laboratories. *Sample $\mathrm{O}_{u} 2$ was not analyzed by Spain 2 lab.

Additional file 5: Table S5. Percentage of classification of the eight olive oils purchased in Italy using results obtained by Organoleptic Evaluation and Chemical Analysis performed on these olive oils by 8 IOC laboratories. * In brackets the percentages of classification according to the chemical analysis without the ethyl esters content are reported.

Additional file 6: Table S6. Percentage of classification of the eight olive oils purchased in USA using results obtained by Panel Test and Chemical Analysis performed on these olive oils by 8 IOC laboratories. *In brackets the percentages of classification according to the chemical analysis without the ethyl esters content are reported.

\section{Abbreviations}

EVOO: extra virgin olive oil; IOC: International Olive Council; ICB: International Certificated Body; FAEE: fatty acid ethyl esters.

\section{Authors' contributions}

All research has been done by the authors. All authors read and approved the final manuscript.

\section{Author details}

${ }^{1}$ Dipartimento di Chimica e Tecnologie del Farmaco, Sapienza Università di Roma, Rome, Italy. ${ }^{2}$ Istituto di Metodologie Chimiche, CNR, Laboratorio di Risonanza Magnetica "Annalaura Segre", Monterotondo, Rome, Italy. ${ }^{3}$ Dipartimento di Farmacia, Università di Napoli "Federico II", Naples, Italy. 


\section{Acknowledgements}

This work was supported by "e-ALIERB: un OPEN LAB per caratterizzare e valorizzare i prodotti alimentari ed erboristici del territorio laziale" Project (Regione Lazio LR13/2008, CUP B82115003570002).

\section{Competing interests}

The authors declare that they have no competing interests.

\section{Availability of data and materials}

The datasets supporting the conclusions of this article are included within the article and its additional files.

\section{Funding}

This work was supported by "e-ALIERB: un OPEN LAB per caratterizzare e valorizzare i prodotti alimentari ed erboristici del territorio laziale" Project (Regione Lazio LR13/2008, CUP B82115003570002).

\section{Publisher's Note}

Springer Nature remains neutral with regard to jurisdictional claims in published maps and institutional affiliations.

Received: 6 February 2017 Accepted: 7 April 2017

Published online: 26 July 2017

\section{References}

1. The Commission of the European Communities. Commission Regulation (EEC) No. 2568/91 on the characteristics of olive oil and oliveresidue oil and on the relevant methods of analysis. Off J Eur Union. 1991:L248:1-102

2. International Olive Oil Council. Sensory analysis of olive oil, method for the organoleptic assessment of virgin olive oil. 2015. IOC/T.20/Doc. No. 15/Rev. 8. http://www.internationaloliveoil.org/estaticos/view/224testing-methods. Accessed 22 Jan 2017.

3. Frankel EN. Nutritional and biological properties of extra virgin olive oil. J Agric Food Chem. 2011;59:785-92.
4. Angerosa F. Sensory quality of olive oils. In: Aparicio R, Harwood J, editors. Handbook of olive oil, analysis and properties. Gaithersburg: Aspen Publishers; 2000. p. 355-92.

5. Salas JJ, Harwood JL, Martínez-Force E. Lipid metabolism in olive: biosynthesis of triacylglycerols and aroma components. In: Aparicio $R$ Harwood J, editors. Handbook of olive oil, analysis and properties. New York: Springer; 2013. p. 97-127.

6. The European Commission. Commission Regulation (EU) No. 432/2012 establishing a list of permitted health claims made on foods, other than those referring to the reduction of disease risk and to children's development and health. Off J Eur Union. 2012;L136:1-40.

7. Camin F, Pavone A, Bontempo L, Wehrens R, Paolini M, Faberi A, Marianella RM, Capitani D, Vista S, Mannina L. The use of IRMS, ${ }^{1} H$ NMR and chemical analysis to characterise Italian and imported Tunisian olive oils. Food Chem. 2016;19:98-105.

8. International Olive Oil Council. List of chemical testing laboratories recognised by the International Olive Council for the period from 1.12.2014 to 30.11.2015. 2014. IOC/T.21/Doc. No. 13/Rev. 17

9. International Olive Oil Council. List of laboratories undertaking the sensory analysis of virgin olive oils recognised by the International Olive Council for the period from 1.12.2014 to 30.11.2015. 2014. IOC/T.28/Doc. No. 3/Rev. 17.

10. Gómez-Coca RB, Moreda W, Pérez-Camino MC. Fatty acid alkyl esters presence in olive oil versus organoleptic assessment. Food Chem. 2012;135:1205-9.

11. Conte L, Mariani C, Gallina Toschi T, Tagliabue S. Alchil esteri e composti correlati in oli d'oliva vergini: loro evoluzione nel tempo. RISG. 2014;91:21-9.

12. International Olive Oil Council. Trade standard applying to olive oils and olive pomace oils. 2016. IOC/T.15/NC No. 3/Rev. 11

13. The European Commission. Commission Delegated Regulation (EU) No. 2016/2095 of 26 September 2016 amending Regulation (EEC) No. $2568 / 91$ on the characteristics of olive oil and olive-residue oil and on the relevant methods of analysis. Off J Eur Union. 2016;L326:1-6.

14. Loreto G, Giansante L, Alfei B, Giacinto L. Alchil esteri ed altri indicatori per la tutela della qualità e della genuinità degli oli extra vergini italiani. RISG. 2014;91:35-45

\section{Submit your manuscript to a SpringerOpen ${ }^{\circ}$ journal and benefit from:}

- Convenient online submission

- Rigorous peer review

- Immediate publication on acceptance

- Open access: articles freely available online

- High visibility within the field

- Retaining the copyright to your article

Submit your next manuscript at $>$ springeropen.com 